\title{
A rat study of the use of end-to-side peripheral nerve repair as a "babysitting" technique to reduce the deleterious effect of chronic denervation
}

\author{
Olawale A. R. Sulaiman, MD, PhD, ${ }^{1}$ and Tessa Gordon, PhD, DSc ${ }^{2}$ \\ 1Department of Neurosurgery, Ochsner Medical Center, New Orleans, Louisiana; and 2Division of Neuroscience, University of \\ Alberta Faculty of Medicine, Edmonton, Alberta, Canada
}

\begin{abstract}
OBJECTIVE Functional recovery is disappointing after surgical repair of nerves that are injured far from their target organs and/or after delayed repair. In the former case, a nerve transfer that transects a distal nerve fascicle to innervate denervated targets is one strategy to promote nerve regeneration and functional recovery. An alternate strategy tested in this study is to perform an end-to-side neurorrhaphy to "babysit" (protect) the denervated distal nerve stump at the time of nerve repair and reduce the deleterious effect of chronic denervation on nerve regeneration.
\end{abstract}

METHODS In the hindlimbs of Sprague-Dawley rats, the common peroneal (CP) nerve was transected unilaterally and the distal CP nerve stump inserted through a perineurial window into the intact tibial (TIB) nerve, i.e., CP-TIB end-to-side neurorrhaphy. In the first experiment, TIB nerve motoneurons that had regenerated and/or sprouted axons into the $\mathrm{CP}$ nerve within 3 months were stimulated to elicit contractions, and thereafter, identified with retrograde dyes for counting. In the second experiment, the intact TIB nerve was transected and cross-sutured to a 3-month chronically denervated distal CP nerve stump that had either been "protected" by ingrown TIB nerves after CP-TIB neurorrhaphy or remained chronically denervated. Thereafter, the number of retrogradely labeled TIB nerve motoneurons that had regenerated their nerves within 3 months were counted and reinnervated tibialis anterior (TA) muscles weighed.

RESULTS A mean ( \pm SE) of $231 \pm 83$ TIB nerve motoneurons grew into the end-to-side CP distal nerve stump with corresponding ankle flexion; $32 \%$ regenerated their axons and $24 \%$ sprouted axons from the intact TIB nerve, eliciting ankle flexor-extensor co-contraction. In the second experiment, after a 3-month period of TIB nerve regeneration, significantly more TIB motoneurons regenerated their axons into "protected" than "unprotected" CP distal nerve stumps within 3 months (mean $332 \pm 43.6$ vs $235 \pm 39.3$ motoneurons) with corresponding and significantly higher numbers of regenerated nerve fibers, resulting in significantly better recovery of reinnervated TA muscle weight.

CONCLUSIONS These experiments in rats demonstrated that delayed nerve repair is more effective when the deleterious effects of chronic denervation of the distal nerve stump are reduced by protecting the nerve stump with ingrowing nerve fibers across an end-to-side insertion of the distal nerve stump into a neighboring intact nerve. Such an end-toside neurorrhaphy may be invaluable as a means of preventing the atrophy of distal nerve stumps and target organs after chronic denervation, which allows for effective reinnervation of the protected distal nerve stumps and target organs over distance and time.

https://thejns.org/doi/abs/10.3171/2018.3.JNS172357

KEYWORDS end-to-side neurorrhaphy; nerve regeneration; peripheral nerve surgery; chronic denervation

$\mathrm{F}$ UNCTIONAL recovery in patients is frequently disappointing, especially after proximal nerve injuries in which regenerating nerves must grow over long distances to reinnervate denervated targets. ${ }^{24}$ This disappointing outcome occurs despite the capacity of Schwann cells (SCs) in the peripheral nervous system to support nerve regeneration after injury. ${ }^{11}$ The slow rate of axonal regeneration of 1-3 $\mathrm{mm}$ /day leaves injured neurons with their axons isolated from target organs (chronic axotomy) and the SCs of the distal nerve stump chronically denervated until axons regenerate through the stump and reinnervate denervated targets. ${ }^{11}$ This may take months to years in patients. In a rat model of nerve injury and surgical repair, we established that chronic axotomy and, to

ABBREVIATIONS AIN = anterior interosseous nerve; $\mathrm{CP}=$ common peroneal; $\mathrm{RAG}=$ regeneration-associated gene; $\mathrm{SC}=\mathrm{Schwann}$ cell; $\mathrm{TA}=$ tibialis anterior; $\mathrm{TIB}=$ tibial. SUBMITTED October 3, 2017. ACCEPTED March 1, 2018.

INCLUDE WHEN CITING Published online September 14, 2018; DOI: 10.3171/2018.3.JNS172357. 
an even greater extent, chronic SC denervation progressively reduces axonal regeneration, ${ }^{11,14,18}$ with evidence that the reduction in neuronal regenerative capacity and SC capacity to support regenerating axons is due to a progressive decline in expression of regeneration-associated genes (RAGs) in the injured neurons and in the denervated SCs. ${ }^{14,21}$ Hence, there is a window of opportunity after injury during which RAGs are maximally upregulated ${ }^{17,21}$ and during which axonal regeneration and reinnervation of muscle targets is fully supported..$^{11,14}$ This period is a maximum of 6 weeks in rats.

There is a clear need for development of strategies to accelerate the rate of axonal regeneration and/or to sustain RAG expression and, in turn, the regenerative capacity of injured peripheral nerves. One such strategy includes the use of brief low-frequency electrical stimulation of the injured neuron, which increases the rate of outgrowth of sensory and motor axons across the injury site and, in turn, significantly accelerated reinnervation of denervated targets in animals and humans. ${ }^{7,8,10,14,15,32,33}$ The electrical stimulation prolongs RAG expression ${ }^{32}$ and is effective in promoting axon regeneration, even after delayed nerve repair. ${ }^{9}$

An alternative surgical strategy is to shorten the distance for nerves to regenerate to their denervated targets using a nerve transfer, namely the dissection and transection of a fascicle from an intact nerve in order to suture the proximal stump of the fascicle to a denervated distal nerve stump close to the denervated target. ${ }^{27}$ While this strategy is frequently used with the view that distal denervated targets undergo irreversible atrophy, an alternate strategy, first implemented a century ago, is to insert the denervated distal nerve stump into the side of a neighboring intact nerve. This is an end-to-side (terminolateral) neurorrhaphy, first described in $1901,{ }^{23}$ which has been used clinically in some cases..$^{3,13,28,38}$ Thus, nerves grow into the distal stump with evidence, primarily from animal studies, of reinnervation of denervated targets $2,5,6,22,26,40,41$ and some evidence of better sensory than motor nerve regeneration. ${ }^{36,44}$ This is a surgical option when a proximal nerve stump is not available, as is frequently the case after root avulsion injuries. ${ }^{13}$ The number of nerve fibers that grow out from the intact donor nerve into the inserted nerve stump is small unless the donor nerve is sufficiently damaged to promote outgrowth of axons into the inserted nerve and,

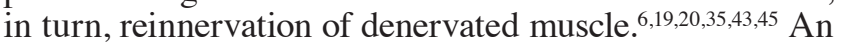
avenue that has not been explored is to use the end-to-side insertion technique to provide nerve fibers from an intact nerve to grow into the denervated nerve stump. These axons may, at least partly, prevent the denervation atrophy of the indwelling SCs in the distal stump, and the denervated target muscle and sense organs, prior to performing a direct nerve repair of the proximal nerve stump to the "protected" distal nerve stump.

The option of protecting the SCs in the chronically denervated distal nerve stump was recently explored in animal experiments when short autologous nerve bridges were placed between a denervated distal nerve stump and a donor nerve. The procedure was effective in protecting the distal nerve stump because there were significantly more neurons that regenerated their axons into a protected than an unprotected nerve stump after delayed nerve repair. ${ }^{16,25}$ Performing an end-to-side neurorrhaphy for protection of the distal nerve stump from SC and target denervation atrophy prior to a nerve repair may also be a viable option to prevent deterioration of the stump's growth environment prior to the surgical repair of the cut nerve stumps.

In this study, we used a rat model of end-to-side neurorrhaphy to determine whether the occupancy of a longterm denervated distal nerve stump by nerve fibers from the donor nerve is an effective strategy to "babysit" the chronically denervated nerve stump and thereby, improve nerve regeneration through the stump. We demonstrated that the intact donor nerve fibers both sprout and regenerate their axons across the neurorrhaphy. Thereby they provide protection to enhance nerve regeneration and target reinnervation after delayed nerve repair.

\section{Methods \\ Animals}

Twenty-seven female Sprague-Dawley rats (180-200 $\mathrm{g}$ each) were utilized in one control $(n=9)$ and two experimental groups (1 and 2) of 9 rats each. All procedures were reviewed and approved by the University of Alberta animal care committee following the Canadian Council of Animal Care guidelines for animal experimentation. Rats were maintained in a standard facility with a 12-hour light/dark cycle as well as ad libitum water and standard rat chow.

\section{Surgical Procedures}

All surgeries were performed in aseptic conditions while rats were under deep general anesthesia induced by intraperitoneal injection of sodium pentobarbital $(45 \mathrm{mg} /$ $\mathrm{kg}$ ). In the anesthetized rats, the skin overlying the lateral thigh of the right hindlimb was incised from the sciatic notch toward the knee. The overlying hamstring muscles were dissected along their fascial boundary to expose the underlying sciatic nerve and its common peroneal (CP), tibial (TIB), and sural nerve branches. In the unoperated left hindlimb, the skin overlying the tibialis anterior (TA) muscle was incised for atraumatic removal of the muscle in the third surgery (see below).

\section{First Surgery}

In the rats in the control group and experimental groups 1 and 2 , the CP nerve in the right hindlimb was transected. In the control group, the two nerve stumps were each tied with 4-0 suture and then sutured to innervated biceps femoris muscle to prevent regeneration of the transected CP nerve fibers for 3 months (Fig. 1A). In experimental groups 1 and 2 , the proximal $\mathrm{CP}$ nerve stump was sutured to innervated muscle while the transected distal CP nerve stump was inserted into the intact TIB nerve via a small window created by cutting through the epineuria and perineuria and thereafter stabilized with Tisseel glue (CP-TIB end-to-side neurorrhaphy; Fig. 1B). The hamstring muscles were sutured together and the skin incision closed with absorbable 4-9 suture. The rats were placed on a heating pad until they recovered before returning them to their cages. 

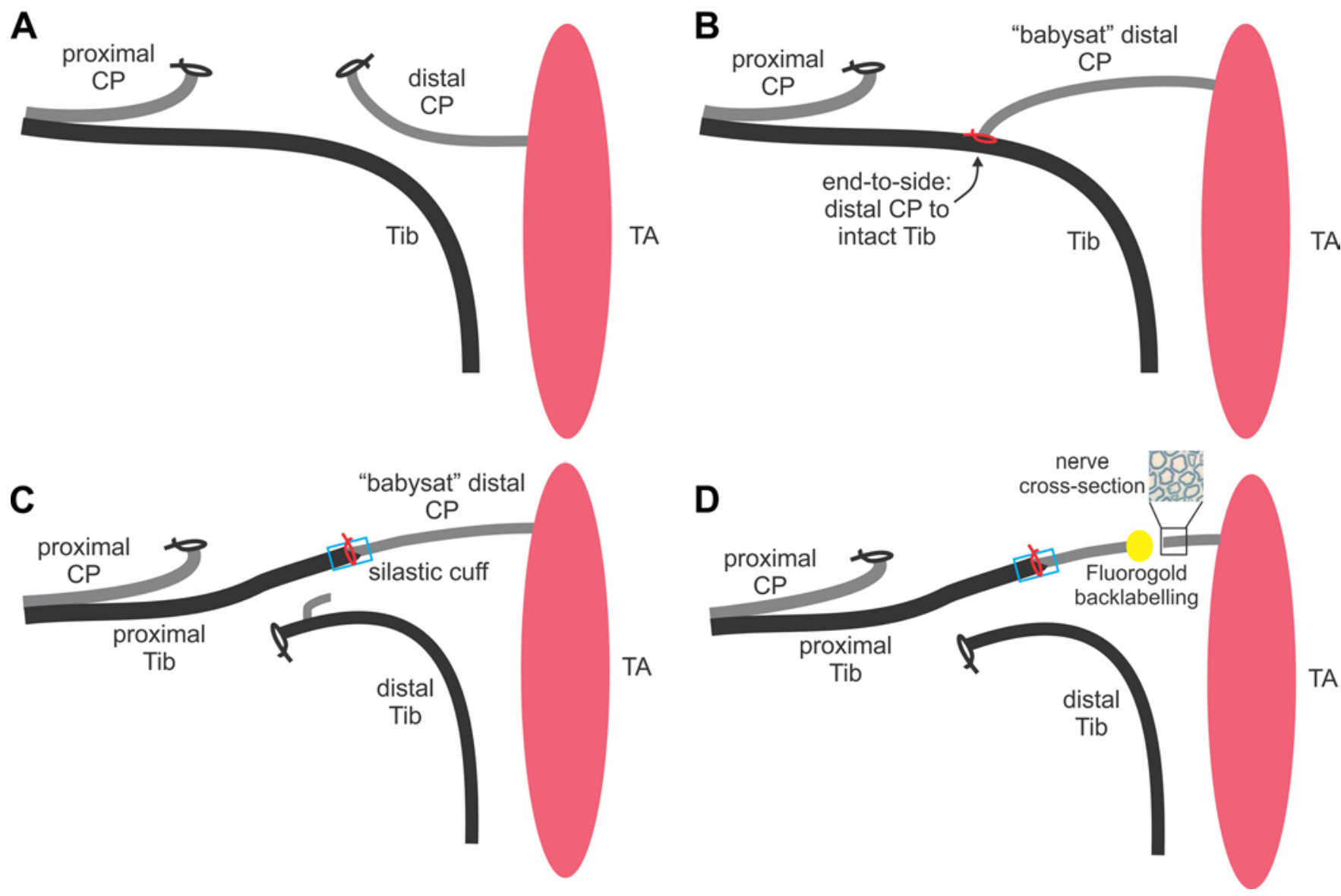

FIG. 1. A: In the first surgery, the CP nerve was transected in the right hindlimb in two groups of rats, the control group and experimental groups 1 and 2. In rats in the control group, the CP nerve stumps were each ligated and then sutured to innervated biceps femoris muscle (not shown) to prevent CP nerve regeneration into the unprotected CP distal nerve stump. B: In rats in experimental groups 1 and 2, the distal CP nerve stump was protected from chronic denervation by inserting the stump end-to-side into the TIB nerve through a perineurial window, i.e., a CP-TIB end-to-side neurorrhaphy. C: The second surgery occurred 3 months later. In rats in experimental group 1 (not shown, see Fig. 2), retrograde dyes were applied to the CP and TIB nerves for enumeration of motoneurons. In rats in the control group, the ligated 3-month chronically denervated CP distal nerve stump was refreshed and the TIB nerve was cut in order to cross-suture the proximal TIB nerve stump to the unprotected 3-month chronically denervated CP distal nerve stump. In experimental group 2, the proximal TIB nerve stump was cross-sutured to the 3-month chronically denervated CP distal stump that had been protected by a CP-TIB end-to-side neurorrhaphy 3 months prior. The TIB distal stump was ligated to prevent reinnervation. D: In the third surgery 3 months later, in the control group and experimental group 2, the CP nerve was transected $20 \mathrm{~mm}$ from the neurorrhaphy site for Fluoro-Gold retrograde labeling of the TIB motoneurons that had regenerated their axons into the CP nerve stump. Figure is available in color online only.

\section{Second Surgery}

In the rats in all groups (control and experimental groups) the second surgery in the right hindlimb was performed 3 months after the first.

In the control group rats, the TIB nerve was cut. Then, the ligated, 3-month, chronically denervated CP distal stump was cut from its insertion into innervated muscle and refreshed by removing $2 \mathrm{~mm}$ from its free end and a TIB-CP cross-suture was performed (Fig. 1C) to encourage TIB nerve regeneration through the chronically denervated $\mathrm{CP}$ distal nerve stump (unprotected $\mathrm{CP}$ nerve).

In the rats in experimental group 1, the $\mathrm{CP}$ nerve distal to its insertion end-to-side into the TIB nerve, the CP-TIB neurorrhaphy, and the TIB nerve distal to the neurorrhaphy were exposed for aseptic and atraumatic electrical stimulation to evaluate muscle target reinnervation. The
$\mathrm{CP}$ and TIB nerves were then transected to apply fluorescent dyes (Fig. 2) to evaluate the number of TIB motoneurons that sent axons into the inserted $\mathrm{CP}$ distal nerve stump and whose nerve fibers remained intact in the TIB nerve distal to the site of the neurorrhaphy performed 3 months previously.

In the rats in experimental group 2, the TIB nerve was cut proximal to the insertion point of the CP-TIB endto-side neurorrhaphy 3 months previously and the distal stump was sutured to adjacent innervated muscle to prevent reinnervation. The $\mathrm{CP}$ distal nerve stump that had been inserted into the side of the TIB nerve as a CP-TIB end-to-side neurorrhaphy for "protection," was cut from the site of the neurorrhaphy and refreshed by removing $2 \mathrm{~mm}$ from its free end. A TIB-CP cross-suture was performed (Fig. 1C) to encourage TIB nerve regeneration 


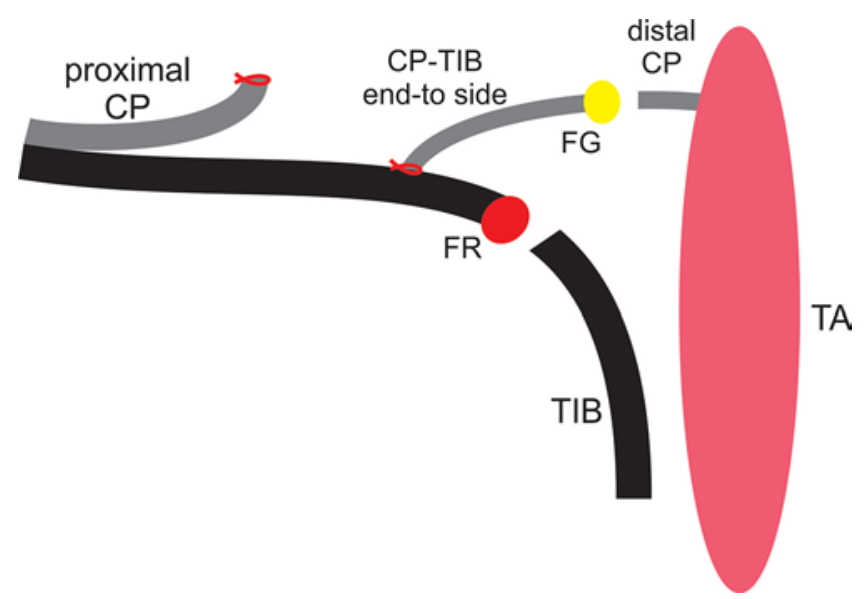

FIG. 2. In the rats in experimental group 1 in which the CP nerve was cut in the first surgery to perform an end-to-side CP-TIB neurorrhaphy (see Fig. $1 \mathrm{~A}$ and $\mathrm{B}$ ), retrograde dyes (Fluoro-Gold [FG] and Fluoro-Ruby [FR]) were applied to the CP nerve $20 \mathrm{~mm}$ from the neurorrhaphy site and to the TIB nerve $10 \mathrm{~mm}$ from the site, respectively, 3 months later. Thereafter, TIB motoneurons that sent axons into the CP distal stump and/or remained within the TIB nerve distal to the neurorrhaphy, respectively, were counted. Figure is available in color online only.

through the "TIB nerve-reinnervated $\mathrm{CP}$ distal nerve stump" (i.e., the "babysat" or protected CP nerve).

Following these second surgeries in all the rat groups, the surgical sites were closed and the rats were allowed to recover from anesthesia before their return to their cages as described above. Note that in all rats, a short 3-mm Silastic tube was used to draw the TIB proximal and CP distal nerve stumps together using a technique that we have previously described. ${ }^{12}$ Briefly, the needle of a 10-0 suture thread was inserted into the middle of the length of the tube to "catch" the epineuria of the proximal and distal nerve stumps before exiting the tube in the middle. Thus, the two nerve stumps were gently brought together by surface tension, a process that allows for excellent apposition of the nerve stumps.

\section{Third Surgery}

In the rats in the control group and experimental group 2 , the $\mathrm{CP}$ nerve in the operated right hindlimb was cut distal to the TIB-CP cross-suture site for 1) removal of the TIB nerve-reinnervated CP stump for histology, 2) atraumatic removal of TA muscle from both hindlimbs for weighing, prior to 3) application of a retrograde dye in the right hindlimb to count TIB motoneurons that had regenerated axons into the unprotected and protected (babysat) $\mathrm{CP}$ distal nerve stumps (Fig. 1D).

\section{Evaluation of Outcomes}

\section{In Situ Muscle Contractions}

In the operated right hindlimb of the rats in experimental group 1, the distal CP nerve stump and the TIB nerve proximal and distal to the CP-TIB end-to-side neurorrhaphy site were exposed for aseptic maximal electrical stimulation to evoke contractions of the ankle flexor muscles (including TA), ankle extensor muscles (including triceps surae), and ankle flexor-extensor co-contractions. A steril- ized bipolar electrode was used and the electrodes were also placed on the corresponding CP and TIB nerve sites in the operated contralateral left hindlimb for stimulation. Using a Grass 9 stimulator, the voltage of a $100-\mu$ sec bipolar electrical pulse was increased at $0.5 \mathrm{~Hz}$ to 2 -times threshold to evoke maximal muscle contractions. The evoked contractile forces of the same muscles in the left unoperated hindlimb were rated as a 5 on a 0 - to 5 -point contraction scale by observing ankle flexion and extension visually followed by palpated isometric force generated by ankle flexion and extension. Thereafter, the bipolar electrodes were moved to the nerves in the operated right hindlimb and the flexor, extensor, and combined muscle contractions evaluated using the same measurements as had been used in the left, previously unoperated hindlimb. No contraction at all was rated as 0 on a 5-point scale, with a score of 5 being the contraction that was equal to that in the left unoperated hindlimb.

Following these qualitative measures of muscle reinnervation, the hamstring muscles were sutured together in the left hindlimb and the incision was closed. In the right hindlimb, the CP and TIB nerves were prepared for application of retrograde dyes as described below.

\section{Retrograde Labeling of Motoneurons}

In the rats in experimental group 1, one of two fluorescent retrograde dyes, i.e., Fluoro-Gold (4\% hydrostilbamidine bis[methanesulphonate]; Sigma-Aldrich no. 39286) and Fluoro-Ruby (5\%, Molecular Probes) diluted in distilled water, was applied to the $\mathrm{CP}$ nerve $20 \mathrm{~mm}$ distal to the CP-TIB end-to-side neurorrhaphy site. The second retrograde dye was applied to the TIB nerve $10 \mathrm{~mm}$ distal to the CP-TIB end-to-side neurorrhaphy in the right hindlimb (Fig. 2). The dyes were alternated between the nerves in different rats. The cut end of each nerve was inserted into a small Silastic pool that was fashioned around the nerve and filled with a dye. A period of 1 hour was used for exposure to the dyes, after which the Silastic pools were removed and the nerves thoroughly irrigated with saline. The wound was closed and the rats were allowed to recover from the anesthesia.

Five days after application of the retrograde dyes, the rats were deeply anesthetized for transfusion of $4 \%$ paraformaldehyde via the aorta. Thereafter, the fixed lumbosacral spinal cord (L3-6) was removed and frozen in order to cut sagittal cryostat sections of 50- $\mu$ m thickness. All Fluoro-Gold and Fluoro-Ruby-stained motoneurons whose nuclei were visible were counted and the numbers corrected with the correction factor of Abercrombie. ${ }^{1}$ The motoneurons stained with either Fluoro-Gold or FluoroRuby were also examined for co-staining, indicative of sprouted axons.

In the rats in the control group (unprotected $\mathrm{CP}$ nerves) and experimental group 2 (protected CP nerves), FluoroGold was applied to the cut CP distal nerve stump $20 \mathrm{~mm}$ from the cross-suture site in the right hindlimb. This application followed the aseptic and atraumatic excision of 1) TA muscles from both hindlimbs, and 2) a 3-mm CP nerve segment distal to the site of cutting the right $\mathrm{CP}$ nerve for Fluoro-Gold application. 


\section{Muscle Weights}

In the rats in the control group and experimental group 2 , the TA muscles were excised from both hindlimbs aseptically in the third surgery (see above), dried on filter paper, and weighed for comparison.

\section{Nerve Histology}

In the rats in the control group and experimental group 2, the 3-mm-long excised distal CP nerves were placed into $3 \%$ glutaraldehyde fixation for cutting semi-thin cross-sections, and undergoing osmium staining, araldite embedding, and toluidine blue staining as described previously. ${ }^{16}$ Myelinated axons were counted throughout the nerve sections. At least four areas of the nerve section were chosen randomly to count the number of myelinated nerves and to measure their nerve fiber cross-sectional areas. The total number of TIB nerve fibers that regenerated into the CP distal nerve stump was calculated from the ratio of the total nerve area and the sum of the areas selected in the section (at least four) and multiplied by the sum of the numbers of nerve fibers counted in the selected areas.

\section{Statistical Analysis}

The numbers of motoneurons whose axons had regenerated and/or sprouted in experimental rat group 1 and whose $\mathrm{CP}$ axons had regenerated in the control group and experimental group 2 were expressed as mean values $( \pm$ $\mathrm{SE}$ ). Both these counts and the CP nerve fiber counts were compared by 1-way ANOVA and Student t-test, and significance was accepted at the 95\% confidence level $(p<0.05)$.

\section{Results}

\section{TIB-CP End-to-Side Neurorrhaphy}

\section{Number of Motoneurons That Regenerated Axons}

In the rats in experimental group 1, the $\mathrm{CP}$ distal nerve stump was inserted into the intact nerve through a perineurial window as a CP-TIB end-to-side neurorrhaphy (Fig. 1B). Three months later, fluorescent dye application (Fig. 2) was used to identify TIB nerve motoneurons that had sent their axons into the inserted CP distal nerve stump and those whose axons in the TIB nerve had "sprouted" into the $\mathrm{CP}$ distal stump. In the example of three TIB motoneurons that were backlabeled from the $\mathrm{CP}$ nerve with Fluoro-Ruby (Fig. 3A left) and two motoneurons that were backlabeled after applying Fluoro-Gold to the TIB nerve distal to the neurorrhaphy (Fig. 3A right), the two outer motoneurons are double-labeled and one in the middle is backlabeled with Fluoro-Ruby only. The double-labeled motoneurons with axons in both CP and TIB nerves were interpreted as those that had sprouted axons into the $\mathrm{CP}$ nerve through the neurorrhaphy while retaining their axons in the TIB nerve. The interpretation of the TIB motoneuron backlabeled with Fluoro-Ruby alone was that the TIB nerve was disrupted by the neurorrhaphy and the TIB motoneuron regenerated its axon into the $\mathrm{CP}$ nerve via the neurorrhaphy.

A mean $( \pm \mathrm{SE})$ of $231 \pm 83$ TIB motoneurons regenerated their axons $20 \mathrm{~mm}$ into the inserted $\mathrm{CP}$ distal nerve stump, a number that was not statistically different from
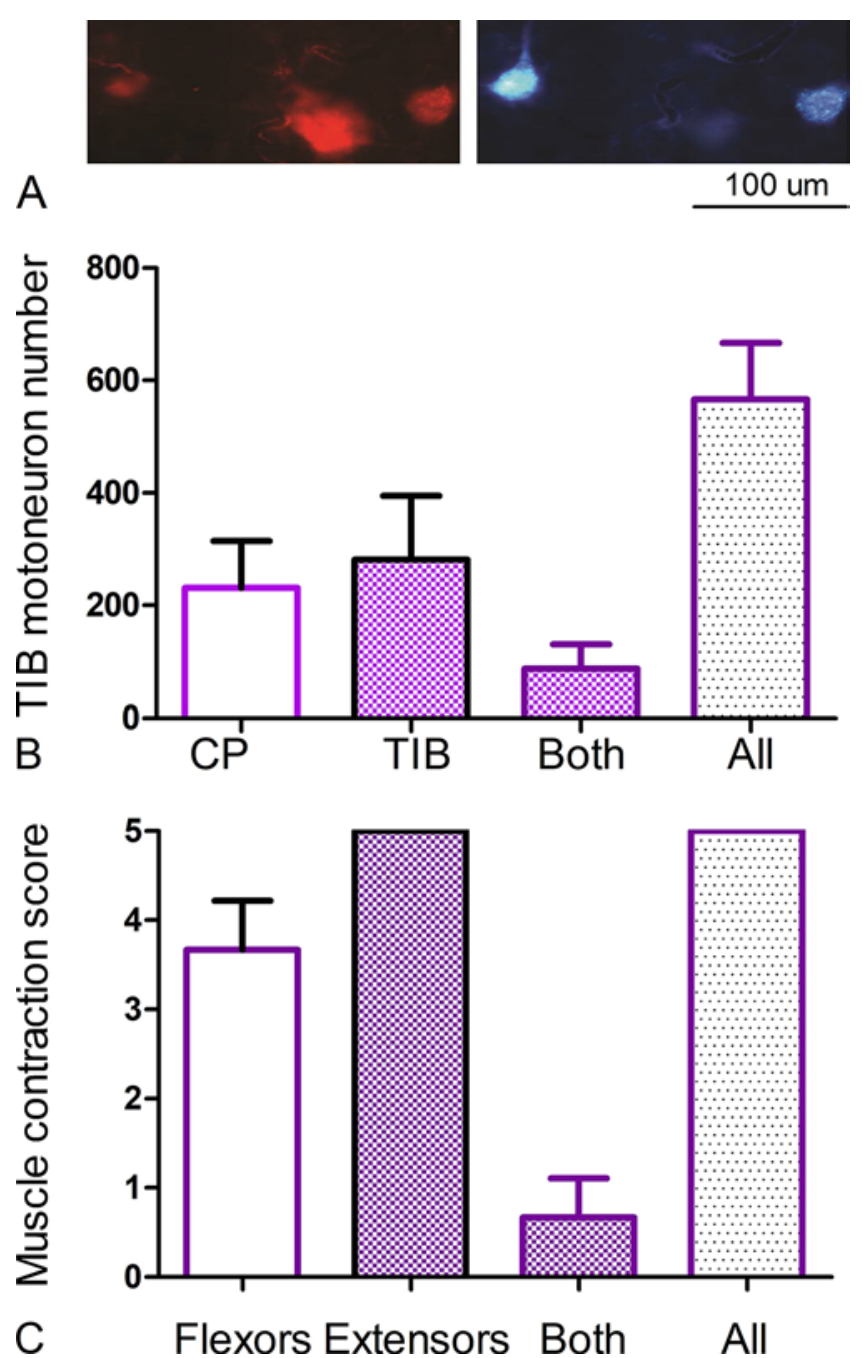

FIG. 3. A: In the rats of the experimental group 1, an example of the dye-filled TIB motoneurons is shown on the left and right micrographs after retrograde application of Fluoro-Ruby to the TIB nerve distal to the CP-TIB end-to-side insertion (neurorrhaphy) and Fluoro-Gold to the inserted distal CP nerve stump, respectively (see Fig. 2). B: The mean $( \pm \mathrm{SE})$ number of TIB motoneurons that were backlabeled from the inserted distal CP nerve stump, the intact TIB nerve, and from both the inserted CP distal nerve stump and the intact TIB nerve, 3 months after the CP-TIB end-to-side insertion of the CP nerve into the intact TIB nerve. The total mean number of TIB motoneurons is also shown with the mean number of TIB motoneurons that supplied the TIB nerve in the contralateral unoperated normal hindlimb. C: The mean $( \pm$ SE) muscle contraction score on a $0-5$ scale for flexor, extensor, and combined muscles that was obtained just prior to the backlabeling of the TIB motoneurons. Figure is available in color online only.

the number remaining in the TIB nerve distal to the $\mathrm{CP}$ end-to-side neurorrhaphy (Fig. 3B). The mean of $55 \pm 7.5$ TIB motoneurons (24\%) that were double-labeled with both the Fluoro-Ruby applied to the TIB nerve and Fluoro-Gold applied to the CP nerve or vice versa, was significantly less than the mean number of TIB motoneurons that regenerated their axons into the inserted CP distal nerve stump or the numbers remaining in the TIB nerve ( $\mathrm{p}<0.5$; Fig. 3B). We concluded that the nerves of the double-labeled TIB motoneurons had sprouted axons into 
the inserted CP nerve stump while sustaining their intact axons in the TIB nerve distal to the site of the CP-TIB insertion.

\section{Reinnervated Muscles}

In the rats of the same experimental group, the strength of muscle contraction of the ankle flexor and extensor muscles was assessed by rating the contractions on a $0-5$ scale, with 0 representing no visible muscle contraction and 5 representing contractions comparable to corresponding muscles in the contralateral hindlimb whose innervation remained intact. Bipolar electrodes were used to electrically stimulate the TIB nerves 1 ) above the CP-TIB endto-side insertion of the denervated CP distal nerve stump, 2) proximal to the insertion, and 3 ) distal to the insertion. Thus, the contractions of 1) flexor, 2) extensor, and 3) both muscle groups were rated; the muscles included the TA, triceps surae, or both muscles, respectively.

The muscle contraction scores (Fig. 3C) mirrored the mean values of 231 and 55 TIB motoneurons whose nerve fibers reinnervated and sprouted to reinnervate the denervated flexor muscles (Fig. 3B). The observed contraction of the flexor muscles in response to the stimulation of the TIB nerves via the end-to-side insertion was not as strong as that of the contralateral intact muscles (Fig. 3C) but corresponded well with the number of TIB motoneurons that regenerated their nerves through the CP-TIB end-to-side neurorrhaphy (Fig. 3B). The remaining TIB motoneurons with axons within the nerve distal to the neurorrhaphy generated extensor muscle contraction that equaled the contralateral muscles, consistent with the remaining mean number of 280 motoneurons (approximately 33\%; Fig. 3B) reinnervating three times as many muscle fibers in the partially denervated extensor muscles to compensate for the reduced innervation. Stimulation of the TIB nerve proximal to the neurorrhaphy evoked a co-contraction of both the flexor muscles and the extensor muscles, the contraction in the flexor muscles being relatively weak (Fig. $3 \mathrm{C})$. This is consistent with the relatively small number of TIB motoneurons sprouting axons from intact TIB nerves through the end-to-side insertion of the $\mathrm{CP}$ nerve into the TIB nerve (Fig. 3B).

\section{TIB-CP Nerve Cross-Suture}

\section{Number of TIB Motoneurons That Regenerated Their Axons}

After a period of 3 months, the chronically denervated CP nerve stump was refreshed in the control rat group $(\mathrm{n}=$ 10) to cross-suture the proximal stump of the freshly transected TIB nerve to a chronically denervated CP nerve stump that was not protected by TIB nerves (unprotected CP nerve, Fig. 4A). In experimental rat group 2, the denervated $\mathrm{CP}$ distal nerve stump was protected (babysat) by TIB axons via a CP-TIB end-to-side neurorrhaphy for 3 months (Fig. 1B), after which the TIB nerve was transected proximal to the neurorrhaphy and cross-sutured to the protected chronically denervated $\mathrm{CP}$ distal nerve stump (protected CP nerve, Fig. 3C). Three months later retrograde dyes were applied distal to the cross-suture site to count and compare the number of TIB motoneurons that had regenerated into the unprotected $\mathrm{CP}$ nerve (control) and protected CP nerve (experimental group 2) (Fig. 1D).
There was a significant increase of $41 \%$ in the number of TIB motoneurons that regenerated their axons through the protected as compared with the unprotected CP distal nerve stump: a mean of $332 \pm 43.6$ motoneurons regenerated through the protected CP nerve as compared to 235 \pm 39.3 motoneurons whose axons regenerated through
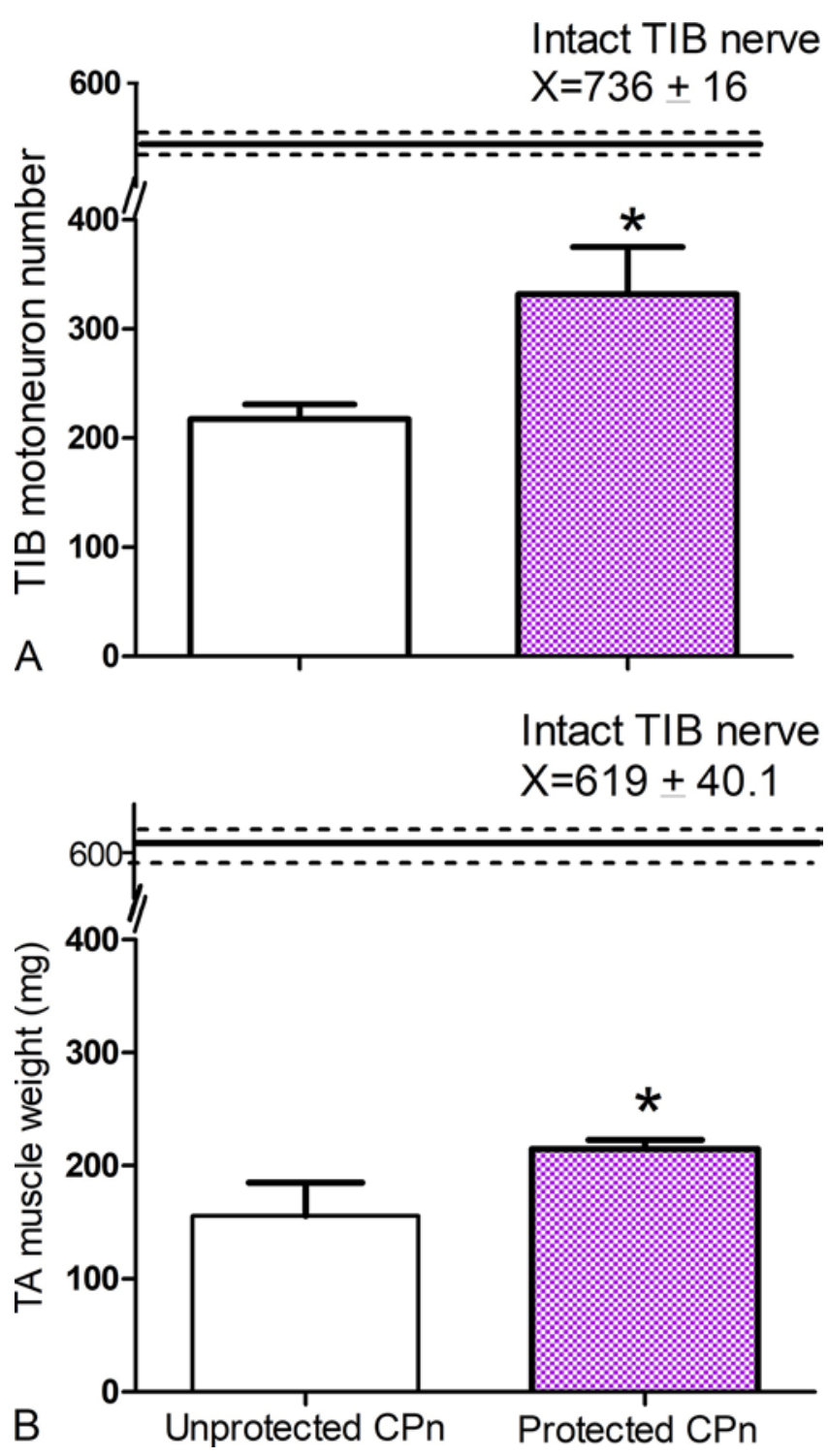

FIG. 4. The mean ( $\pm \mathrm{SE}$ ) of the number of TIB motoneurons that were backlabeled from the distal $C P$ nerve stump $(A)$, and $(B)$ the weight of reinnervated TA muscles 3 months after a TIB-CP cross-suture repair of the transected TIB nerve and a 3-month chronically denervated CP distal nerve stump that was either protected by TIB axons after CP-TIB end-to-side neurorrhaphy (Protected CPn) or not protected (Unprotected $\mathrm{CPn}$ ). The protection was achieved by inserting the $\mathrm{CP}$ distal nerve stump into the intact TIB nerve, a CP-TIB end-to-side neurorrhaphy. The number of TIB motoneurons regenerating nerves into the protected $\mathrm{CP}$ nerve, and the TA muscle weights after protection, were significantly greater than when the CP nerve was not protected $(p<0.05)$. Nonetheless, the number of regenerated TIB motoneurons and the TA muscle weights did not attain the mean $( \pm \mathrm{SE})$ of the numbers of motoneurons with axons in the intact TIB nerve or the weights of the normally innervated TA muscles in the contralateral unoperated normal hindlimb (horizontal lines). Figure is available in color online only. 
the unprotected $\mathrm{CP}$ nerve, a difference that was statistically significant $(\mathrm{p}<0.5$; Fig. $4 \mathrm{~A})$. Note that the increased number of motoneurons regenerating their axons through the protected CP nerve stump is approximately $50 \%$ of the mean of $736 \pm 16$ TIB motoneurons that supply the nerve in the contralateral unoperated hindlimb. Hence, the protection is partial and does not fully compensate for the chronic denervation of the CP nerve stumps (Fig. 4A).

\section{Reinnervated Muscles}

The reinnervated TA muscles were significantly heavier when the TIB motoneurons regenerated their axons through the protected chronically denervated $\mathrm{CP}$ distal nerve stumps as compared to those that regenerated through the unprotected CP nerve stumps (Fig. 4B). In both cases, the regenerating TIB motor nerves reinnervated denervated TA muscles. Recovery was not complete, however, as the reinnervated TA muscle weights were significantly lower than the contralateral control muscle weights. The incomplete recovery is likely because there were fewer nerves regenerating into the muscle (normal counts being $736 \pm 16$ ) and because the regenerating nerves likely had not yet reinnervated the muscles and/or the newly reinnervated muscles had not yet recovered their former size after denervation atrophy.

\section{Number of Regenerated Axons}

There was an obvious increase in the number of myelinated axons but not their size or extent of myelination in the protected compared with the unprotected $\mathrm{CP}$ distal nerve stumps after TIB-CP nerve cross-suture and regeneration for 3 months (Fig. 5). The mean numbers of regenerated nerves that were counted in the protected as compared with the unprotected $\mathrm{CP}$ distal nerve stumps were 3717 \pm 558 and $1653 \pm 999$, respectively. The total area occupied by the nerves that corresponded to these values was $399,450 \pm 12,915$ and $206,033 \pm 16,109 \mu \mathrm{m}^{2}$, respectively. These differences in the number of regenerating nerves and the total area occupied by the nerves were statistically significant $(\mathrm{p}<0.05)$. The numbers of TIB nerve fibers regenerating into the $\mathrm{CP}$ distal nerve stumps were $38 \%$ and $17 \%$, respectively, of the normal numbers of myelinated nerve fibers in the unoperated rat tibial nerve of $9900 .{ }^{31}$ The approximately 2 -fold increase in the number of regenerated TIB nerve fibers in the protected $\mathrm{CP}$ distal nerve stumps is greater than the 1.4-fold increase in the number of TIB motoneurons that regenerated their nerves into the nerve stump after the TIB-CP cross-suture, 3 months previously (Fig. 4A); the latter were $45 \%$ and $32 \%$, respectively. This difference may be accounted for by the higher number of sensory neurons regenerating their axons into the $\mathrm{CP}$ nerve stumps when protected.

\section{Discussion}

One of the key factors that limits functional recovery after severe nerve damage is the time-dependent loss of the growth-permissive environment in the denervated dis-

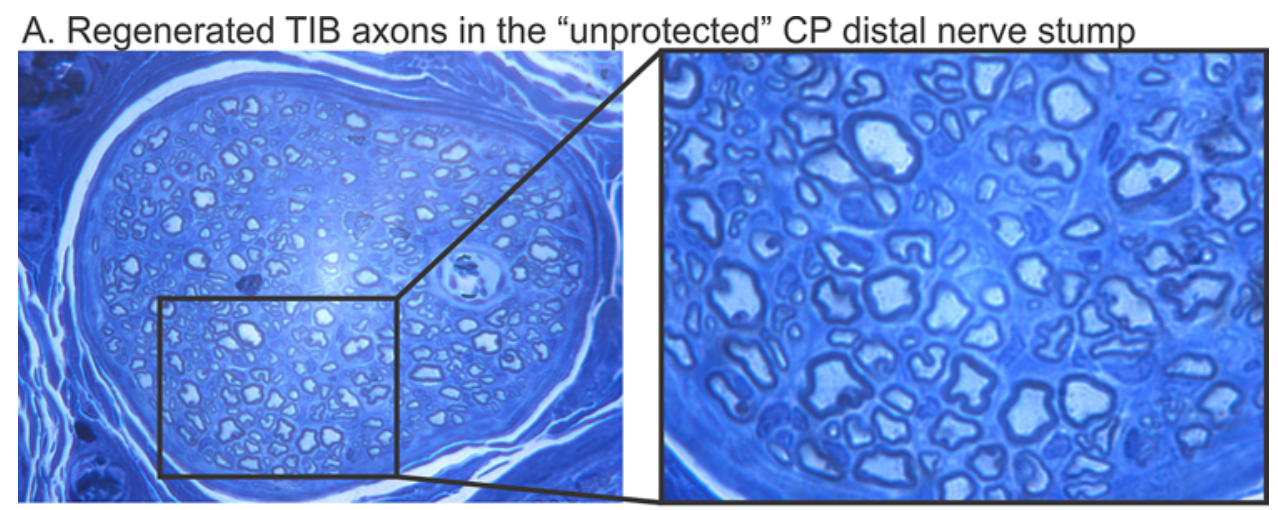

B. Regenerated TIB axons in the "protected" CP distal nerve stump

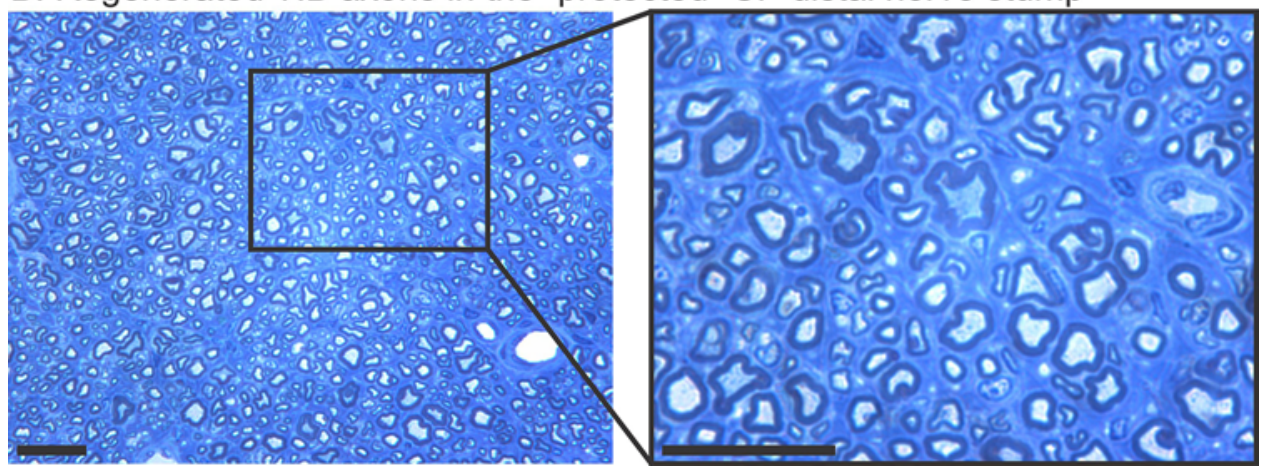

FIG. 5. Toluidine blue-stained, 3-month regenerated TIB nerve fibers in the CP distal nerve stump after cross-suture of the freshly transected TIB proximal nerve stump to a 3-month chronically denervated CP distal nerve stump that remained denervated without protection $(A)$ or was protected by TIB axons after inserting the CP distal nerve stump into the side of the intact TIB nerve (CP-TIB end-to-side neurorrhaphy; B). Bar = $20 \mu \mathrm{m}$. Figure is available in color online only. 
tal nerve stump that is provided by the SCs. ${ }^{11}$ This study explored the possibility of extending the time window of permissive nerve growth by occupying the chronically denervated distal nerve stump with axons derived through a perineurial window from an intact nerve in a rat model of end-to-side neurorrhaphy. This was achieved by inserting the transected CP distal nerve stump end-to-side into the window in the intact TIB nerve to encourage growth of TIB nerve fibers into the CP nerve stump, thereby conferring protection against chronic denervation of all the SCs in the CP distal nerve stump (experimental group 2; Fig. $1 \mathrm{~B}$ and $\mathrm{C}$ ). A considerable number of TIB motoneurons were found to regenerate their axons through a perineurial window into the inserted $\mathrm{CP}$ nerve stump to reinnervate flexor muscles, while relatively few sprouted their axons (Fig. 3B), resulting in a corresponding small co-contraction of flexor and extensor muscles (Fig. 3C). The remaining TIB nerve fibers were left intact distal to the site of the end-to-side neurorrhaphy. They reinnervated all the denervated muscle fibers in the partially denervated extensor muscles, resulting in normal contractile strength of the muscles (Fig. 3C). The TIB axons that had regenerated and sprouted into the 3-month chronically denervated CP distal nerve stump conferred protection to the chronically denervated SCs within the 3-month chronically denervated CP distal nerve stump. The evidence was that: 1) significantly more TIB motoneurons regenerated their axons when the TIB proximal nerve stump was cross-sutured to the protected CP distal nerve stump in experimental group 2 as compared to those that regenerated their axons into the unprotected chronically denervated CP nerve stump in the control rat group (Fig. 4A); 2) there were significantly more myelinated nerve fibers in the protected as compared with the unprotected CP distal nerve stumps; and 3) the reinnervated TA muscles recovered significantly more of their weight after the TIB nerves regenerated through protected as compared to unprotected CP nerve stumps (Fig. 4B).

\section{Effective Outgrowth of Motor Fibers Into an End-to-Side Nerve Insertion}

The end-to-side surgical procedure used in this study is the one that has been adopted widely by surgeons to anastomose a transected piece of gut to another piece of gut to avoid the mismatch of transected intestinal portions. ${ }^{4}$ Examples include the surgical cross-suture of the ileum to the colon after a hemicolectomy or the suture of a small to a large loop of small intestine. The procedure has also been used for repair of blood vessels under conditions such as occlusive disease and for the creation of arteriovenous fistulas. ${ }^{29}$ The technique was first described for nerves in 1873 but it fell into disrepute when poor clinical outcomes were reported. ${ }^{37}$ Its adoption by Viterbo in the $1990 \mathrm{~s}^{38-42}$ restored interest in the procedure, especially in the context of regaining function when the proximal nerve stump was unavailable but a healthy neighboring donor nerve could provide axons. ${ }^{3}$ The question of whether or not to open an epineurial window in the donor nerve prior to suturing the denervated distal nerve stump was addressed by several surgeons, including Viterbo and Lundborg, with the conclusion that the window was unnecessary. ${ }^{26,40}$ The issue of whether to open epineurial windows remains controversial, however, with recent evidence from animal studies that an epineurial window is essential for donor nerves to grow into an inserted nerve stump. ${ }^{6,16}$ Under conditions in which 3-9 nerve autografts were inserted between an intact TIB donor nerve and a recipient CP distal nerve stump in a rat hindimb, the windows were essential for ingrowth of axons from the donor nerve, and most of the nerve fibers regenerated out from the donor nerve rather than sprouted axons into the nerve autografts. ${ }^{16,25}$ Indeed, sprouted axons were the exception rather than the rule. In this study of an end-to-side neurorrhaphy in the rat, we transected the perineurium in creating a window for insertion of the distal CP nerve stump into the recipient intact TIB nerve. Under these conditions, an average of approximately $24 \%$ TIB motoneurons sprouted axons into the denervated $\mathrm{CP}$ nerve stump (Fig. 3B).

The percentage of TIB motoneurons whose axons grew into the CP nerve stump after the CP-TIB end-to-side insertion was approximately $32 \%$ (Fig. 3A). This proportion corresponds exactly with the proportion of TIB motoneurons that regenerated their axons into the denervated $\mathrm{CP}$ nerve stump through three perineurial windows of $500-\mu \mathrm{m}$ radius via three cross-bridges into a denervated $\mathrm{CP}$ distal nerve stump. ${ }^{16}$ The size of the perineurial window in the current study was the same size as in our former study in which we established a linear relationship between the number of motoneurons regenerating axons through the side-to-side cross-bridges and the size of the perineurial windows cut into the donor TIB and the recipient $\mathrm{CP}$ nerves.

The mean value of $231 \pm 83$ TIB motoneurons whose axons grew into the end-to-side inserted CP distal nerve stump (Fig. 3A) is not significantly different from that of $335 \pm 86$ TIB motoneurons that regenerated their axons across three side-to-side cross-bridges and grew in both directions into a denervated CP distal nerve stump in our former study. ${ }^{16}$ In the former study, the number of sensory neurons that had regenerated their axons was also reported with the numbers of motor and sensory neurons corresponding to approximately $32 \%$ and $37 \%$ of the total number of TIB motor and sensory neurons of 900 and 8900, respectively. ${ }^{31}$

Following end-to-side neurorrhaphy in this study and insertion of side-to-side bridges in our past study, there were approximately $33 \%$ of remaining donor nerve fibers supplying their extensor muscles. That these partially denervated muscles fully recovered their contractile strength (Fig. 3C) is consistent with the ability of these nerve fibers that retain their functional contact with extensor muscle fibers to extend (sprout) nerve fibers from the last node of Ranvier or beyond this node, and to reinnervate all the denervated muscle fibers. ${ }^{34}$ The capacity of nerves with intact nerve-muscle contacts to increase the number of muscle fibers that they supply in partially denervated muscles is 5- to 8-fold, sufficient to reinnervate all denervated muscle fibers unless the partial denervation exceeds $80 \%-85 \% .^{30,34}$

\section{More Motoneurons Regenerate Their Axons Through a Protected Distal Nerve Stump After Delayed Nerve Repair}

The differences between this study of end-to-side neu- 

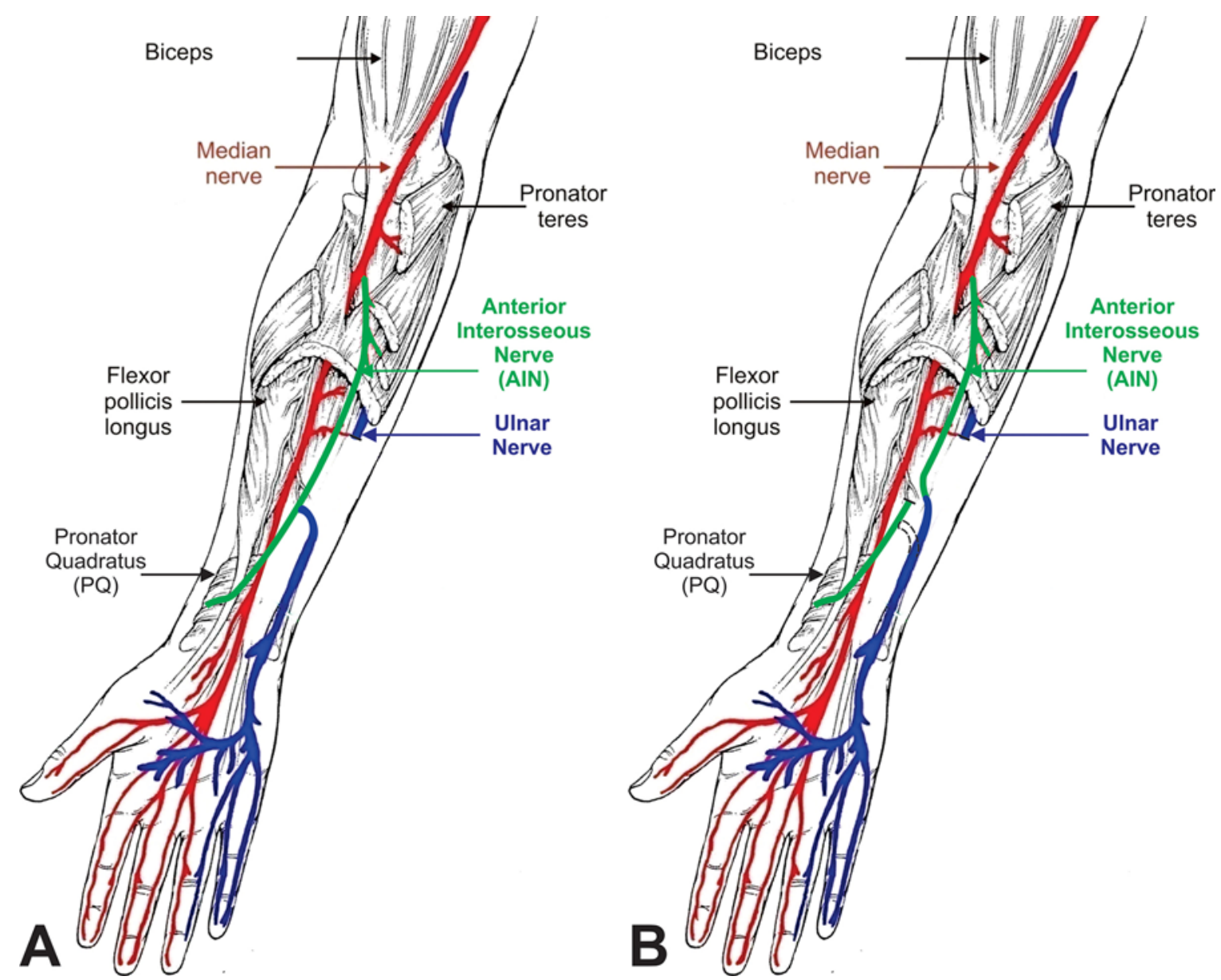

FIG. 6. Illustration of the use of an end-to-side neurorrhaphy. In this example, the distal stump of the transected ulnar nerve is inserted end-to-side into the AIN branch of the median nerve (A) to protect the denervated distal ulnar nerve stump and its denervated target hand muscles, including the first dorsal interosseous muscle, from denervation atrophy. Thereafter (B), the proximal AIN stump is cross-sutured to the protected distal ulnar nerve, a nerve transfer from the AIN proximal to the neurorrhaphy to the protected distal ulnar nerve. Copyright Tessa Gordon. Published with permission. Figure is available in color online only.

rorrhaphy and side-to-side bridging in our earlier study were: 1) the denervated CP distal nerve stump was inserted in an end-to-side fashion (Figs. 1B and 2) in the present study, in contrast to the placement of side-to-side crossbridges that were inserted between the intact donor TIB nerve and the recipient denervated CP distal nerve stump; and 2) only a single perineurial window was opened in the present study, in contrast to the three windows opened in the former study. The protection offered by the TIB axons within the CP distal nerve stump over a 3-month period, while significantly improving nerve regeneration, was inferior to that provided by the TIB axons that entered the distal CP stump via three side-to-side cross-bridges:16 $100 \%$ of the $\mathrm{CP}$ motoneurons regenerated their axons into the protected $\mathrm{CP}$ distal nerve stump after suturing the proximal $\mathrm{CP}$ nerve stump to the distal $\mathrm{CP}$ nerve stump that had been protected by three cross-bridges..$^{16}$ In contrast, approximately $45 \%$ of the TIB motoneurons regenerated their axons into the protected $\mathrm{CP}$ distal nerve stump with significantly improved reinnervated muscle weight in the present study (Fig. 4A). This comparison indicates that the bridging technique with three inserted cross-bridges allows for better nerve regeneration after protection. Despite this finding, however, the end-to-side technique that has been used when the original proximal nerve stump is not available for the end-to-end suture of proximal and distal nerve stumps, is a valid technique for protecting chronically denervated distal nerve stumps and, in turn, encouraging the regeneration of axons through the protected nerve stump. The end-to-side neurorrhaphy technique may be particularly valuable for repair of proximal nerve injuries where the distance of regeneration is long and regeneration is confounded by the chronic denervation of the distal nerve stump and the target organs. Under the conditions of an injury to the ulnar nerve in the upper forearm that cannot be retrieved after major trauma, such as a shark bite for example, an end-to-side neurorrhaphy of the ulnar nerve distal stump to the side of the intact anterior interosseous nerve (AIN) branch of the median nerve would provide babysitting (protection) to the denervated distal ulnar nerve and to its denervated muscle targets in the hand (Fig. 6 ). Once the patient is medically stable, the AIN is cut to perform end-to-end transfer of the AIN to the distal ulnar nerve stump that had been protected by nerve fibers from the AIN. Thereby, the AIN fibers would be encouraged to regenerate through the protected distal ulnar nerve stump 
into the hand and, in turn, to reinnervate the denervated muscles and skin.

The technique of using end-to-side neurorrhaphy followed by surgical repair is superior to the surgery of the side-to-side bridging with autologous nerve bridges because of the confounding functional consequence of the common nerve source of the reinnervated muscles and the original muscle in the latter surgery. However, this problem of co-contraction of both muscles may also occur with end-to-side neurorrhaphy.

\section{Conclusions}

Our experiments in rats demonstrated that delayed nerve repair is more effective when the chronic denervation of the distal nerve stump is allayed by protecting the nerve stump with ingrowing nerve fibers across an endto-side insertion of the distal nerve stump into a neighboring intact nerve (a neurorrhaphy). The regeneration was not enhanced to the levels recorded after insertion of cross-bridges between a donor nerve and a recipient denervated nerve stump. However, the technique of inserting cross-bridges requires the availability of a proximal nerve stump. The end-to-side neurorrhaphy, on the other hand, is a viable technique to promote nerve regeneration after delayed nerve repair under circumstances in which the proximal nerve stump is unavailable. In addition, the technique may be invaluable as a means of preventing the atrophy of the distal nerve stumps and the target organs after chronic denervation and, in turn, allowing for the effective reinnervation of the protected distal nerve stumps and target organs over distance and time.

\section{Acknowledgments}

We thank our technician, Mr. Neil Tyreman, for his invaluable assistance with the experiments, Kasra Tajdaran for his drawing of Fig. 6, and the Canadian Institutes for Medical Research for their grant support for these experiments. Tessa Gordon was an Alberta Heritage Foundation for Medical Research Senior Scientist at the time of the experiments.

\section{References}

1. Abercrombie M: Estimation of nuclear population from microtome sections. Anat Rec 94:239-247, 1946

2. Bersaneti JA, Viterbo F, Jorge J, Denadai R: Muscle reinnervation in one or two stages? Experimental study in rats with end-to-side nerve graft. Acta Cir Bras 27:841-847, 2012

3. Bertelli JA, Ghizoni MF: Nerve repair by end-to-side coaptation or fascicular transfer: a clinical study. J Reconstr Microsurg 19:313-318, 2003

4. Biasutti S, Dart AJ, Dart CM, Uquillas E, Jeffcott LB: Endto-side anastomosis of the left ventral colon to the small colon in a neonatal foal with segmental agenesis of the large colon. Aust Vet J 95:217-219, 2017

5. Bontioti E, Kanje M, Lundborg G, Dahlin LB: End-to-side nerve repair in the upper extremity of rat. J Peripher Nerv Syst 10:58-68, 2005

6. Brenner MJ, Dvali L, Hunter DA, Myckatyn TM, Mackinnon SE: Motor neuron regeneration through end-to-side repairs is a function of donor nerve axotomy. Plast Reconstr Surg 120:215-223, 2007

7. Brushart TM, Hoffman PN, Royall RM, Murinson BB, Witzel C, Gordon T: Electrical stimulation promotes motoneuron regeneration without increasing its speed or conditioning the neuron. J Neurosci 22:6631-6638, 2002

8. Brushart TM, Jari R, Verge V, Rohde C, Gordon T: Electrical stimulation restores the specificity of sensory axon regeneration. Exp Neurol 194:221-229, 2005

9. Elzinga K, Tyreman N, Ladak A, Savaryn B, Olson J, Gordon $\mathrm{T}$ : Brief electrical stimulation improves nerve regeneration after delayed repair in Sprague Dawley rats. Exp Neurol 269:142-153, 2015

10. Foecking EM, Fargo KN, Coughlin LM, Kim JT, Marzo SJ, Jones KJ: Single session of brief electrical stimulation immediately following crush injury enhances functional recovery of rat facial nerve. J Rehabil Res Dev 49:451-458, 2012

11. Fu SY, Gordon T: The cellular and molecular basis of peripheral nerve regeneration. Mol Neurobiol 14:67-116, 1997

12. Furey MJ, Midha R, Xu QG, Belkas J, Gordon T: Prolonged target deprivation reduces the capacity of injured motoneurons to regenerate. Neurosurgery 60:723-733, 2007

13. Geuna S, Papalia I, Tos P: End-to-side (terminolateral) nerve regeneration: a challenge for neuroscientists coming from an intriguing nerve repair concept. Brain Res Brain Res Rev 52:381-388, 2006

14. Gordon T: Electrical stimulation to enhance axon regeneration after peripheral nerve injuries in animal models and humans. Neurotherapeutics 13:295-310, 2016

15. Gordon T, Amirjani N, Edwards DC, Chan KM: Brief postsurgical electrical stimulation accelerates axon regeneration and muscle reinnervation without affecting the functional measures in carpal tunnel syndrome patients. Exp Neurol 223:192-202, 2010

16. Gordon T, Hendry M, Lafontaine CA, Cartar H, Zhang JJ, Borschel GH: Nerve cross-bridging to enhance nerve regeneration in a rat model of delayed nerve repair. PLoS One 10:e0127397, 2015

17. Gordon T, Tetzlaff W: Regeneration-associated genes decline in chronically injured rat sciatic motoneurons. Eur J Neurosci 42:2783-2791, 2015

18. Gordon T, Tyreman N, Raji MA: The basis for diminished functional recovery after delayed peripheral nerve repair. J Neurosci 31:5325-5334, 2011

19. Haninec P, Kaiser R, Dubový P: A Comparison of collateral sprouting of sensory and motor axons after end-to-side neurorrhaphy with and without the perineurial window. Plast Reconstr Surg 130:609-614, 2012

20. Hayashi A, Pannucci C, Moradzadeh A, Kawamura D, Magill C, Hunter DA, et al: Axotomy or compression is required for axonal sprouting following end-to-side neurorrhaphy. Exp Neurol 211:539-550, 2008

21. Höke A, Redett R, Hameed H, Jari R, Zhou C, Li ZB, et al: Schwann cells express motor and sensory phenotypes that regulate axon regeneration. J Neurosci 26:9646-9655, 2006

22. Kale SS, Glaus SW, Yee A, Nicoson MC, Hunter DA, Mackinnon SE, et al: Reverse end-to-side nerve transfer: from animal model to clinical use. J Hand Surg Am 36:16311639.e2, 2011

23. Kennedy R: On the restoration of coordinated movement after nerve-crossing, with interchange of function of the cerebral cortical centers. Philos Trans R Soc Lond B Biol Sci 194B:127-163, 1901

24. Kline DG, Hudson AR: Nerve Injuries: Operative Results for Major Nerve Injuries, Entrapments and Tumors. Philadelphia: Saunders, 1995

25. Ladak A, Schembri P, Olson J, Udina E, Tyreman N, Gordon T: Side-to-side nerve grafts sustain chronically denervated peripheral nerve pathways during axon regeneration and result in improved functional reinnervation. Neurosurgery 68:1654-1666, 2011

26. Lundborg G, Zhao Q, Kanje M, Danielsen N, Kerns JM: Can sensory and motor collateral sprouting be induced from 
intact peripheral nerve by end-to-side anastomosis? J Hand Surg Br 19:277-282, 1994

27. Mackinnon SE: Donor distal, recipient proximal and other personal perspectives on nerve transfers. Hand Clin 32:141151,2016

28. Millesi H, Schmidhammer R: End-to-side coaptation-controversial research issue or important tool in human patients. Acta Neurochir Suppl (Wien) 100:103-106, 2007

29. Numajiri T, Fujiwara T, Nishino K, Sowa Y, Uenaka M, Masuda S, et al: Double vascular anastomosis for safer free jejunal transfer in unfavorable conditions. J Reconstr Microsurg 24:531-536, 2008

30. Rafuse VF, Gordon T, Orozco R: Proportional enlargement of motor units after partial denervation of cat triceps surae muscles. J Neurophysiol 68:1261-1276, 1992

31. Schmalbruch H: Fiber composition of the rat sciatic nerve. Anat Rec 215:71-81, 1986

32. Sharma N, Marzo SJ, Jones KJ, Foecking EM: Electrical stimulation and testosterone differentially enhance expression of regeneration-associated genes. Exp Neurol 223:183191,2010

33. Sharma N, Moeller CW, Marzo SJ, Jones KJ, Foecking EM: Combinatorial treatments enhance recovery following facial nerve crush. Laryngoscope 120:1523-1530, 2010

34. Tam SL, Archibald V, Jassar B, Tyreman N, Gordon T: Increased neuromuscular activity reduces sprouting in partially denervated muscles. J Neurosci 21:654-667, 2001

35. Tarasidis G, Watanabe O, Mackinnon SE, Strasberg SR, Haughey BH, Hunter DA: End-to-side neurorraphy: a longterm study of neural regeneration in a rat model. Otolaryngol Head Neck Surg 119:337-341, 1998

36. Tarasidis G, Watanabe O, Mackinnon SE, Strasberg SR, Haughey BH, Hunter DA: End-to-side neurorrhaphy resulting in limited sensory axonal regeneration in a rat model. Ann Otol Rhinol Laryngol 106:506-512, 1997

37. Tos P, Colzani G, Ciclamini D, Titolo P, Pugliese P, Artiaco S: Clinical applications of end-to-side neurorrhaphy: an update. BioMed Res Int 2014:646128, 2014

38. Viterbo F, Amr AH, Stipp EJ, Reis FJ: End-to-side neurorrhaphy: past, present, and future. Plast Reconstr Surg 124 (6 Suppl):e351-e358, 2009

39. Viterbo F, Romão A, Brock RS, Joethy J: Facial reanimation utilizing combined orthodromic temporalis muscle flap and end-to-side cross-face nerve grafts. Aesthetic Plast Surg 38:788-795, 2014
40. Viterbo F, Teixeira E, Hoshino K, Padovani CR: End-to-side neurorrhaphy with and without perineurium. Sao Paulo Med J 116:1808-1814, 1998

41. Viterbo F, Trindade JC, Hoshino K, Mazzoni A: Two endto-side neurorrhaphies and nerve graft with removal of the epineural sheath: experimental study in rats. Br J Plast Surg 47:75-80, 1994

42. Viterbo F, Trindade JC, Hoshino K, Mazzoni Neto A: Endto-side neurorrhaphy with removal of the epineurial sheath: an experimental study in rats. Plast Reconstr Surg 94:10381047, 1994

43. Yan JG, Matloub HS, Sanger JR, Zhang LL, Riley DA, Jaradeh SS: A modified end-to-side method for peripheral nerve repair: large epineurial window helicoid technique versus small epineurial window standard end-to-side technique. J Hand Surg Am 27:484-492, 2002

44. Yang W, Yang J, Yu C, Gu Y: End-to-side neurotization with different donor nerves for treating brachial plexus injury: an experimental study in a rat model. Muscle Nerve 50:67-72, 2014

45. Zhang Z, Soucacos PN, Bo J, Beris AE: Evaluation of collateral sprouting after end-to-side nerve coaptation using a fluorescent double-labeling technique. Microsurgery 19:281-286, 1999

\section{Disclosures}

The authors report no conflict of interest concerning the materials or methods used in this study or the findings specified in this paper.

\section{Author Contributions}

Conception and design: both authors. Acquisition of data: both authors. Analysis and interpretation of data: both authors. Drafting the article: both authors. Critically revising the article: Gordon. Reviewed submitted version of manuscript: both authors. Approved the final version of the manuscript on behalf of both authors: Gordon. Statistical analysis: Gordon.

\section{Correspondence}

Tessa Gordon: Peter Gilgan Centre for Research and Learning, The Hospital for Sick Children, Toronto, ON, Canada. tessat.gordon@gmail.com. 